\title{
Alocação de Potência em Redes de Comunicação Móveis de Múltiplo Acesso: Aspectos de Implementação via Modelos de Verhulst e Perron-Frobenius
}

\section{Power Allocation in Multiple Access Networks: Implementation Aspects via Verhulst and Perron-Frobenius Models}

\author{
Fábio Engel de Camargo ${ }^{1}$; Mario Lemes Proença $\mathrm{Jr}^{2}$; Taufik Abrão ${ }^{3}$
}

\begin{abstract}
Resumo
Neste trabalho, o modelo de Verhulst e o teorema de Perron-Frobenius são aplicados ao problema de controle de potência em redes de comunicação de múltiplo acesso móveis limitadas por interferência. Analisa-se o compromisso desempenho-complexidade de ambos os algoritmos de alocação de potência (PCA - power control algorithm), bem como são enfatizados aspectos relativos aos custos computacionais inerentes à possibilidade de implementação desses dois métodos no modo distribuído (DPCA - distributed PCA). Como prova-de-conceito, a implementação dos algoritmos é realizada em uma plataforma DSP comercial de ponto flutuante. Os resultados em termos de número de ciclos e tempo de processamento indicam a) viabilidade de implementação do PCA-Verhulst em sistemas celulares $2 \mathrm{G}$ e 3G; b) elevado custo computacional para o PCA-Perron-Frobenius.
\end{abstract}

Palavras-chave: PCA. Controle de potência. DSP. Perron-Frobenius. Verhulst.

\begin{abstract}
In this work, the Verhulst model and the Perron-Frobenius theorem are applied on the power control problem which is a concern in multiple access communication networks due to the multiple access interference. This paper deals with the performance versus complexity tradeoff of both power control algorithm (PCA), as well as highlights the computational cost aspects regarding the implementability of distributed PCA (DPCA) version for both algorithms. As a proof-of-concept the DPCA implementation is carried out deploying a commercial point-floating DSP platform. Numerical results in terms of DSP cycles and computational time as well indicate a) feasibility of implementing the PCA-Verhulst model in $2 \mathrm{G}$ and $3 \mathrm{G}$ cellular systems; b) high computational cost for the PCA-Perron-Frobenius model.
\end{abstract}

Key words: PCA. Power control. DSP. Perron-Frobenius. Verhulst.

\footnotetext{
${ }^{1}$ Aluno de mestrado do Departamento de Ciência da Computação, Universidade Estadual de Londrina; fabioengel@gmail.com.br.

${ }^{2}$ Docente do Departamento de Computação, Universidade Estadual de Londrina; Proença@uel.br

${ }^{3}$ Docente do Departamento de Engenharia Elétrica da Universidade Estadual de Londrina - DEEL-UEL; taufik@uel.br.
} 


\section{Introdução}

Neste trabalho são consideradas redes de comunicação de múltiplo acesso limitadas por interferência uniportadoras, i.e., sistemas do tipo DS/CDMA (direct sequence / code division multiple access). O CDMA é uma técnica de acesso múltiplo de compartilhamento do espectro de frequências baseada no espalhamento espectral dos sinais, ou seja, é um sistema que permite aos usuários transmitirem informações no mesmo intervalo de tempo e compartilharem simultaneamente o mesmo espectro de frequências. Com a técnica CDMA de espalhamento por sequência direta, a informação no domínio do tempo é multiplicada por uma sequência de espalhamento, conhecida também pelo receptor, cuja largura de banda é muito maior que aquela da informação. Isto permite ao receptor realizar a detecção com uma determinada imunidade à interferência causada pelos demais usuários ocupando a mesma faixa espectral, obtendo-se simultaneamente segurança/privacidade na transmissão (HAYKIN; MOHER, 2008).

Ao compartilhar o espectro de frequências no mesmo instante, os usuários do sistema CDMA são expostos ao problema conhecido como efeito nearfar. Este efeito ocorre quando usuários transmitem com mesma potência, mas no entanto aqueles transmissores localizados próximos à unidade receptora, acarretarão níveis de interferência muito mais elevados na recepção em relação àqueles sinais enviados pelos transmissores localizados a distâncias mais longas. Para contornar este problema, os sistemas e redes CDMA requerem a implementação de um rigoroso controle de potência. Assim, para se ter garantia de que todos os sinais cheguem à unidade receptora com o mesmo nível de potência, os transmissores mais distantes são obrigados a elevar seus níveis de potência, contribuindo para um aumento geral do nível de consumo de potência do sistema.
No contexto do link reverso, o objetivo principal do controle de potência consiste em estabelecer níveis de potências, de tal forma que a relação sinal interferência mais ruído (SINR - signal-tointerference plus noise ratio) de cada usuário atinja um limite necessário para um desempenho aceitável. Este limite pode variar, dependendo do nível de desempenho (máxima taxa de erro tolerável) e qualidade de serviço (mínima QoS) requeridos.

Considerando ainda a mobilidade dos usuários, e as mudanças de condições do canal, espera-se que o controle de potência seja realizado por diversas vezes a cada período da informação digital ${ }^{4}$. Para isto, técnicas de controle de potência eficientes, e de baixo custo computacional são necessárias, como as revisadas em (ULLAH; RAIHAN; BARY, 2011) onde algoritmos iterativos de controle de potência distribuídos são utilizados para estimar o vetor de potência ótimo do sistema. Além da utilização de algoritmos iterativos, outras abordagens são utilizadas, como em (DONMEZ, 2011), onde o problema de controle de potência é modelado como um jogo não cooperativo no qual os usuários buscam maximizar sua própria função utilidade sem prejudicar os demais usuários do sistema.

Neste trabalho, dois modelos de controle de potência foram avaliados através da implementação em plataforma DSP de ponto flutuante com o objetivo de determinar os seus respectivos custos computacionais. O primeiro deles, baseado em um método analítico, provê a solução ótima para o problema, sendo caracterizado por uma condição imposta pelo teorema de Perron-Frobenius (BAPAT; RAGHAVAN, 1997; SENETA, 2006). O segundo é um algoritmo iterativo, baseado no modelo populacional de Verhulst (VERHULST, 1838) sendo adaptado para o problema de controle de potência em (GROSS; ABRÃO; JESZENSKY, 2005, 2010). Assim, a contribuição deste trabalho consiste em: a) análise comparada do compromisso

\footnotetext{
${ }^{4}$ No padrão DS/CDMA de segunda geração, a taxa de atualização do controle de potência é de 800 vezes/seg. Enquanto que para DS/CDMA 3G, esta taxa é dobrada.
} 
desempenho-complexidade de dois algoritmos de controle de potência disponíveis na literatura; b) desenvolvimento de aspectos relativos ao custo computacional inerentes à implementação de métodos de controle de potência nos modos distribuído e centralizado; neste sentido, o artigo apresenta a prova-de-conceito de viabilidade a partir da implementação dos dois algoritmos de controle de potência em uma plataforma DSP comercial de ponto flutuante.

O restante deste trabalho está organizado da seguinte forma: A fundamentação matemática do modelo de controle de potência é introduzida na Seção 2. Na Seção 3 e 4 são enunciados os problema de controle de potência na forma analítica e através do modelo de Verhulst, respectivamente. Os resultados numéricos são descritos na Seção 5 e na Seção 6 é analisada a complexidade dos PCAs propostos. Finalmente, as conclusões são feitas na Seção 7 e os aspectos de implementação das estratégias de alocação de recursos em redes de múltiplo acesso móveis são brevemente tratados no Apêndice A.

\section{Modelo de Sistema e Formulação do Problema de Controle de Potência}

Para a construção de cenários típicos em redes CDMA, foi considerado um ambiente com $n$ transmissores e $n$ receptores, classificados de $1, \ldots, n$, capazes de transmitir níveis de potência diferente de zero, $P_{1}, \ldots, P_{\mathrm{n}} \in\left[P_{\min } ; P_{\max }\right]$, os quais são considerados as variáveis do problema de controle de potência; $P_{\text {max }}$ indica o nível máximo de potência que pode operar cada transmissor. Cada receptor $i$ recebe o sinal enviado pelo respectivo transmissor $i$. Entretanto, transmissor e receptor não necessitam ser transmissores e receptores físicos; eles podem, por exemplo, fazer referência ao mesmo receptor físico, no entanto, com diferentes canais em frequência, em tempo, códigos ou feixe em uma antena. Neste trabalho, serão consideradas redes operando com única antena no transmissor e receptor (SISO), bem como diferentes códigos de espalhamento, cada um identificando um único usuário móvel ativo na rede.

Definindo $|h i j|^{2}$ como o ganho de canal (módulo) entre o $j$-ésimo transmissor para o $i$-ésimo receptor, a energia do sinal no ésimo receptor é $T|h i j|^{2} P i$ e a energia de interferência no $i$-ésimo receptor é definida pelo somatório dos sinais interferentes:

$$
T \sum_{k \neq i}\left|h_{i k}\right|^{2} P_{k}
$$

sendo $T$ o período de símbolo ou frame no qual estão sendo avaliados os sinais de todos os usuários ativos simultaneamente ocupando a mesma banda do espectro eletromagnético, em [Hz].Adicionalmente, a potência do ruído AWGN (Additive white Gaussian noise) inerente ao sistema de comunicação é denotada por $\sigma_{\mathrm{i}}^{2}=N_{0} \mathrm{~W}[\mathrm{~W}]$, sendo $N_{0}$ a densidade espectral de potência unilateral de ruído. Isto nos possibilita definir a relação sinal-interferência mais ruído (SINR - signal to interference plus noise ratio) para o $i$-ésimo par receptor/transmissor, ponderada pelo ganho de processamento $F_{i}$.

$$
\gamma_{i}=F_{i} \frac{\left|h_{i i}\right|^{2} P_{i}}{\sigma_{i}^{2}+\sum_{k \neq i}\left|h_{i k}\right|^{2} P_{k}}, \quad i=1,2, \ldots, n
$$

Para uma adequada formulação do problema de otimização de potência, faz-se necessário a definição de uma SINR de limiar, $y_{i}^{\text {th }}$, a qual garante uma mínima condição de SINR para o estabelecimento da comunicação com a mínima qualidade de serviço (QoS - quality of service), i.e., máxima taxa de erro de bit (ou símbolo, ou ainda quadro) tolerável, entre os parâmetros que caracterizam a QoS. Assim, a seguinte condição deve ser garantida:

$$
\gamma_{i} \geq \gamma_{i}^{t h}, \quad i=1,2, \ldots, n
$$

Do ponto de vista da SINR, existem diferentes métricas para a determinação do ponto de operação do sistema. Pode-se determinar a SINR de operação do sistema como sendo aquela que maximiza a eficiência energética (EE), a qual geralmente resulta maior que a SINR de limiar, responsável 
por garantir a mínima QoS, i.e. $\gamma_{\mathrm{EE}}^{\text {opt }}>\gamma^{\text {th }}$ (MIAO; HIMAYAT; LI, 2010; SOUZA et al., 2012). No entanto, está fora do escopo deste trabalho analisar as diferentes métricas para a determinação da SINR ótima. Sendo assim, será adotado uma SINR de limiar típica capaz de garantir a mínima QoS. Para uma discussão apropriada sobre as diferentes métricas na determinação da SINR de operação do sistema, recomenda-se (MIAO; HIMAYAT; LI, 2010; SOUZA et al., 2012; RODRIGUEZ, 2003; SARAYDAR; MANDAYAM; GOODMAN, 2002; GOODMAN; MANDAYAN, 2000).

Em particular, para sistemas CDMA com portadora única, o vetor de potência ótimo, que minimiza a soma total de potência transmitida, será aquele o qual tornará as desigualdades de (3) em igualdades (ULUKUS; YATES, 1998). Esta conclusão é válida também para redes de múltiplo acesso multiportadoras, como por exemplo, redes de comunicação baseadas no princípio de multiplexação por divisão de frequência ortoganal (OFDM), combinado ao DS/CDMA, gerando o sistema MC-CDMA. No entanto, por simplicidade de exposição, neste trabalho serão consideradas apenas redes CDMA uniportadoras. Porém, ressalte-se que em ambos os tipos de redes, uni ou multiportadora, o teorema de Perron-Frobenius torna-se de grande importância a fim de comprovar as condições de existência e unicidade da solução ótima para o problema do controle de potência a ser considerado.

\section{Modelo Analítico para o Problema do Controle de Potência}

De modo conveniente, podemos adequar as inequações de (3) em um formato matricial, a fim de determinar o vetor de potência ótimo de modo analítico. Para isto, estabelecemos

$$
G_{i j}=\left\{\begin{array}{cc}
\frac{\left|h_{i j}\right|^{2}}{F_{i}\left|h_{i i}\right|^{2}} & i \neq j \\
0 & i=j
\end{array}, \quad q_{i}=\frac{\sigma_{i}^{2}}{F_{i}\left|h_{i i}\right|^{2}}\right.
$$

e substituímos em (3) obtendo

$$
\gamma_{i}^{t h}\left(G_{i j} p_{k}+q\right) \leq p_{i}
$$

Desta maneira, o problema primordial de alocação de potência pode ser escrito na forma matricial como (PILLAI; SUEL; CHA, 2005):

$$
\begin{gathered}
\boldsymbol{\Gamma}(\mathbf{G P}+\mathbf{q}) \leq \mathbf{P} \\
\operatorname{com} \boldsymbol{\Gamma}=\left(\begin{array}{ccc}
\gamma_{1}^{t h} & \cdots & 0 \\
& \ddots & \\
0 & & \gamma_{n}^{t h}
\end{array}\right), \mathbf{G}=\left(\begin{array}{ccc}
0 & \cdots & G_{1 n} \\
& \ddots & \\
G_{n 1} & \cdots & 0
\end{array}\right), \\
\mathbf{P}=\left(\begin{array}{c}
P_{1} \\
\vdots \\
P_{n}
\end{array}\right), \mathrm{e} \mathbf{q}=\left(\begin{array}{c}
q_{1} \\
\vdots \\
q_{n}
\end{array}\right) .
\end{gathered}
$$

Assumindo $\mathbf{A}=\boldsymbol{\Gamma} \mathbf{G}$ e $\boldsymbol{\Gamma q}=\mathbf{b}$, então temos

$$
(\mathbf{I}-\mathbf{A}) \mathbf{p} \geq \mathbf{b}
$$

Por definição temos que o vetor de potência que transforma as $n$ inequações de (3) em igualdades também minimiza a soma das potências transmitidas. Isto nos permite calcular a potência ótima:

$$
\mathbf{p}_{\mathrm{o}}=(\mathbf{I}-\mathbf{A})^{-1} \mathbf{b}
$$

com A consistindo em uma matriz regular não negativa com a diagonal principal igual a zero. A existência de uma solução positiva $\mathbf{p}$ para cada b, estará condicionada ao fato de que todos os elementos da inversa de (I-A) sejam positivos para todo $\mathbf{A} \geq 0$, i.e.:

$$
(\mathbf{I}-\mathbf{A})^{-1} \geq 0
$$

Do teorema de Perron-Frobenius (SENETA, 2006; BAPAT; RAGHAVAN, 1997) segue que (8) será verdadeiro se e somente se o módulo do raio espectral de $\mathbf{A}$, definido aqui por $\rho(\mathbf{A})$, for estritamente menor que 1 :

$(\mathbf{I}-\mathbf{A})^{-1} \geq 0$ se e somente se $\rho(\mathbf{A})=\left|\lambda_{\max }(\mathbf{A})\right|<1$

Entretanto, o procedimento de verificação de (9), através da determinação dos autovalores de $\mathbf{A}$, tornase custoso à medida que cresce o grau do polinômio característico da matriz em questão. Por este motivo, optou-se neste trabalho pelo uso do Método da Potência (MP) (SALKUYEH; TOUTOUNIAN, 
2009). O MP consiste em aproximações sucessivas sobre o maior autovalor em módulo de uma matriz quadrada, desprezando os cômputos dos demais autovalores, desnecessários para o nosso problema.

Portanto, adicionalmente ao método analítico aplicado ao problema de controle de potência, têm-se o custo empregado na determinação da viabilidade do método, uma vez que as condições impostas, como o estado momentâneo do canal e a $\gamma^{\text {th }}$, podem inviabilizar a determinação de um vetor de potência ótimo em (7). Como alternativa, tem-se os métodos iterativos, como por exemplo, o modelo de controle de potência de Verhulst (GROSS; ABRÃO; JESZENSKY, 2010), os quais buscam uma aproximação do vetor de potência que satisfaz o problema em questão.

\section{Modelo de Controle de Potência de Verhulst}

O modelo descrito por Verhulst permite que se possa fazer previsões bastante acuradas sobre a evolução temporal do número de indivíduos de uma determinada espécie (VERHULST, 1838). O modelo de Verhulst é dado por:

$$
\dot{p}=z(p)=p\left(1-\frac{p}{p^{*}}\right)
$$

As condições impostas pelo ambiente natural, como disponibilidade de espaço físico e alimentos consideradas no termo $p^{*}$, impedem que uma espécie em um dado instante, denotado por $p(t)$ tenha um crescimento ilimitado.

Este modelo foi adaptado de forma a se tornar aplicável ao controle de potência em redes de comunicação de múltiplo acesso móveis (GROSS; ABRÃO; JESZENSKY, 2005, 2010). Para isto, basta integrar (10) analiticamente, obtendo-se:

$$
p(t)=\frac{\exp (t) p(0) p^{*}}{p^{*}+p(0)[\exp (t)-1]}
$$

No qual o comportamento assintótico é dado por:

$$
\lim _{t \rightarrow \infty} p(t)=p^{*} \quad \forall p(0)>0
$$

Neste caso, se a condição inicial $p(0)$ for maior que $p^{*}$, a primeira derivada de (11) $p$ será sempre negativa, e então $\mathrm{p}(\mathrm{t})$ decrescerá. Caso contrário, onde $p(0)$ é menor que $p^{*}$, a primeira derivada de (11) será positiva e $\mathrm{p}(\mathrm{t})$ crescerá em direção ao seu valor assintótico (GROSS; ABRÃO; JESZENSKY, 2010).

Supondo a necessidade da análise simultânea de diversas espécies, no nosso caso, o controle de potência de todos os usuários, podemos reescrever (10) na forma matricial:

$$
\dot{\mathbf{p}}=z(\mathbf{p})=\mathbf{p} \otimes\left(\mathbf{u}-\mathbf{p} \oslash \mathbf{p}^{*}\right)
$$

sendo $\otimes$ e $\oslash$ o operador matricial ponto-a-ponto de multiplicação e divisão, respectivamente, com $\mathbf{p}=\left[p_{1}, p_{2}, \ldots, p_{\mathrm{u}}\right]^{T}, \mathbf{u}=[1,1, \ldots, 1]^{T}, \mathbf{p}^{*}=\left[p_{1}{ }^{*}, p 2^{*}, \ldots, p^{*} \mathrm{u}\right]^{T}$

e $U$ o número de usuários. Assumindo que o vetor de potência evolui da mesma forma que $\mathbf{p}$ no modelo biológico original de Verhulst, e que estará suficientemente próximo de $\mathbf{p}^{*}$ após $n$ iterações seguidas, então podemos reescrever a partir de (6), admitindo (I-A) $p=b$, a condição de equilíbrio:

$$
\mathbf{p}^{*}=\mathbf{A p}+\mathbf{b}
$$

Aplicando (14) em (13) obtemos

$$
\dot{\mathbf{p}}=\mathbf{p} \otimes[\mathbf{u}-\mathbf{p} \oslash(\mathbf{A p}+\mathbf{b})],
$$

Portanto, a função $\mathbf{z}(\cdot)$ resulta em:

$$
z(-(\mathbf{I}-\mathbf{A}) \mathbf{p}+\mathbf{b})=\mathbf{p} \otimes[\mathbf{u}-\mathbf{p} \oslash(\mathbf{A p}+\mathbf{b})]
$$

Deste modo, o formato de $\mathrm{z}(\cdot)$ dependerá da matriz $\mathbf{A}$ e do vetor $\mathbf{b}$. A fim de discretizar a equação (15), aplica-se o método de integração numérica de Euler, obtendo-se (GROSS; ABRÃO; JESZENSKY, 2010) 


$$
\begin{aligned}
\mathbf{p}(k+1) & =\mathbf{H}(\mathbf{p}[k]) \\
& =\alpha \mathbf{p}[k] \otimes\{\mathbf{u}-\mathbf{p}[k] \oslash[\mathbf{A p}[k]+\mathbf{b}]\}
\end{aligned}
$$

sendo $\mathbf{H}(\cdot)=\left[H_{1}(\cdot) H_{2}(.) \ldots H_{\mathrm{u}}(\cdot)\right]$ as funções de mapeamento, na formato vetorial, dimensão $U$, nas quais, aplicando-se a cada um dos $K$ termos do vetor de equações em (15) resulta:

$$
\begin{aligned}
H_{i}(\mathbf{p}[k]) & =(1+\alpha) p_{i}[k]-\frac{\alpha}{\gamma_{i}^{t h}} \mu_{i}(\mathbf{p}) p_{i}^{2}, \\
& =(1+\alpha) p_{i}[k]-\alpha\left[\frac{\gamma_{i}[k]}{\gamma_{i}^{t h}}\right] p_{i}[k]
\end{aligned}
$$

com

$$
\mu_{i}(\mathbf{p})=\frac{\left|h_{i i}\right|^{2}}{\sigma_{i}^{2}+\sum_{i \neq j}^{k}\left|h_{i j}\right|^{2} p_{j}}
$$

A equação matricial em (17) é equivalente ao seguinte conjunto de equações escalares (GROSS; ABRÃO; JESZENSKY, 2010)

$$
p_{i}(k+1)=(1+\alpha) p_{i}[k]-\alpha\left[\frac{\gamma_{i}[k]}{\gamma_{i}^{t h}}\right] p_{i}[k], \quad i=1, \ldots, U
$$

sendo $\mathrm{p}_{\mathrm{i}}[k]$ e $\mathrm{p}_{\mathrm{i}}(K+1)$ as potências do $i$-ésimo usuário nas iterações $k$ e $k+1$, respectivamente; $\gamma_{\mathrm{i}}[\mathrm{k}]$ é a SINR do usuário na $k$-ésima iteração; $\gamma_{i}^{\text {th }}$ é a mínima condição de SINR para o usuário $i$, a qual garante a mínima qualidade de serviço. $\mathrm{O}$ coeficiente de aceleração, $\alpha €[0,1]$ fixo ou adaptativo (SAMPAIO, 2010), é responsável pela taxa de convergência do algoritmo. Adotando-se um fator $\alpha$ adaptativo obtém-se uma aceleração na taxa de convergência do algoritmo à custa de um incremento marginal de complexidade.

\section{Resultados Numéricos}

\section{Parâmetros de Simulação}

Para a construção do cenário de simulação, considerou-se um ambiente de comunicação unicelular com geometria de célula circular, de área $\mathrm{A}=\pi d$ max $^{2}$, com a $\mathrm{BS}$ posicionada no centro da célula e os usuários móveis distribuídos uniformemente ao redor da BS, sendo $d_{i}$ a distância Euclidiana entre o $i$-ésimo usuário móvel e a BS. Neste cenário foram ignorados o sombreamento e as interferências das células adjacentes. $\mathrm{O}$ termo de atenuação ou desvanecimento de pequena escala do canal foi assumido um processo Gaussiano complexo circularmente simétrico:

$$
h_{i j}=\left(\nu_{i j}+g_{i j} \sqrt{-1}\right) \frac{1}{\sqrt{2}}
$$

sendo $\mathbf{v}_{\mathrm{ij}}$ e $\mathrm{g}_{\mathrm{ij}}$ processos Gaussianos independentes e identicamente distribuídos, i.e, ambos seguem uma distribuição $\sim \mathrm{N}\left(\mu, \sigma^{2}\right)$, de média $\mu=0$ e variância $\sigma^{2}=\mathrm{d}_{\mathrm{ij}}^{-1}$, sendo $\mathrm{d}_{\mathrm{ij}}$ a distância Euclidiana entre o receptor $i$ e o transmissor $j$. Como consequência, a amplitude do processo de desvanecimento de pequena escala $\left|h_{i j}\right|$ será caracterizada por uma distribuição estatística de Rayleigh, descrevendo portanto um canal de comunicação sem linha de visada e não-seletivo em frequência.

Ademais, nas simulações, a SINR mínima a ser garantida foi pré-determinada para diferentes faixas de usuários. A Tabela 1 exibe os valores adotados, bem como os demais parâmetros utilizados nas simulações computacionais, tendo em vista analisar o desempenho e a complexidade de ambos os PCAs iterativos, baseados nos modelos de Verhulst e no Método da Potência. 
Tabela 1 - Parâmetros de Modelo, Sistema e Canal Utilizados

\section{Parâmetro}

\section{Valores}

\section{DS-CDMA}

SINR mín., para [2; 5] usuários

SINR mín., para [6;10] usuários

SINR mín., para [11; 14] usuários

Faixa de Potência de Transmissão

Potência de ruído

Ganho de processamento

Distância máxima entre BS e MT

Tipo de Canal

$$
\begin{aligned}
& \gamma^{\text {th }}=3,16 \cong 5(d B) \\
& \gamma^{\text {th }}=1,60 \cong 2(d B) \\
& \gamma^{\text {th }}=1,12 \cong 0.5(d B) \\
& P_{\text {min }}=0 ; P_{\text {max }}=600[\mathrm{~mW}] \\
& \sigma_{i}^{2}=2 \times 10^{-13}[\mathrm{~mW}], \forall i \\
& \mathrm{~F}_{i}=15, \quad i=1, \ldots, U \\
& d_{\text {max }}=1000 \mathrm{~m}
\end{aligned}
$$

NLOS: Rayleigh plano

\section{Modelo de Verhulst}

Coeficiente de aceleração

Número máximo de iterações

$$
\alpha=0,8
$$

$I_{\max }{ }^{v} C[0,150]$

Modelo Analítico - Método da Potência

\begin{tabular}{ll}
\hline Tolerância para convergência & $\epsilon=10^{-5}$ \\
Número máximo de iterações & $\mathrm{I}_{\max }=15$
\end{tabular}

Fonte: Os autores

\section{Simulação na Plataforma DSP TMS320C6713}

Tendo como objetivo a implementação dos dois modelos na plataforma DSP, optou-se por emular as condições de canal a partir da ferramenta MATLAB instalada em um computador pessoal. No DSP, ocorreu o processamento dos algoritmos de controle de potência sob análise. Desta forma, a cada nova realização, as condições de canal são atualizadas (amostras) seguindo a distribuição estatística em (21), para cada usuário ativo. Esta nova condição de canal é então enviada para a plataforma DSP, que por sua vez, realiza os cálculos e retorna ao MATLAB o resultado do vetor de potência ótimo obtido pelo algoritmo. Ressalte-se que as mesmas condições de canal foram aplicadas aos dois algoritmos.

Devido às restrições de processamento no DSP, que podem resultar em perda do sincronismo na troca de informações com o MATLAB, optou-se por realizar 100 realizações para cada cenário sob análise. Para avaliar a qualidade das soluções do PCA de Verhulst, utilizou-se a figura de mérito erro quadrático médio normalizado (NMSE - normalized mean square error):

$$
\mathrm{NMSE}_{\mathrm{v}}[k]=\left\langle\frac{\left\|p_{\mathrm{v}}[k]-p_{\mathrm{o}}[k]\right\|^{2}}{\left\|p_{\mathrm{o}}[k]\right\|^{2}}\right\rangle, \quad k=1, \ldots, \mathcal{I}_{\max }
$$

sendo $p_{v}$ a potência estimada pelo algoritmo Verhulst e $p_{0}$ a potência ótima, obtida analiticamente via (7), admitindo-se que o problema de alocação de problema apresenta solução; $\|\cdot\|^{2}$ representa a distância Euclidiana quadrática; $<>>$ é o operador média temporal. Resultados para o NMSE de Verhulst, considerando $U \in[2 ; 14]$ com valores 
distintos de SINR mínima, $\gamma^{\text {th }}$ (por faixa de número de usuários) e número de iterações na faixa de $K$ $€[0 ; 150]$ são mostrados na Fig. 1 .

Figura 1 - NMSE para PCA de Verhulst: a) em função do número de usuários e do número de iterações; b) número fixo de iterações, $k=\mathrm{I}=70$.

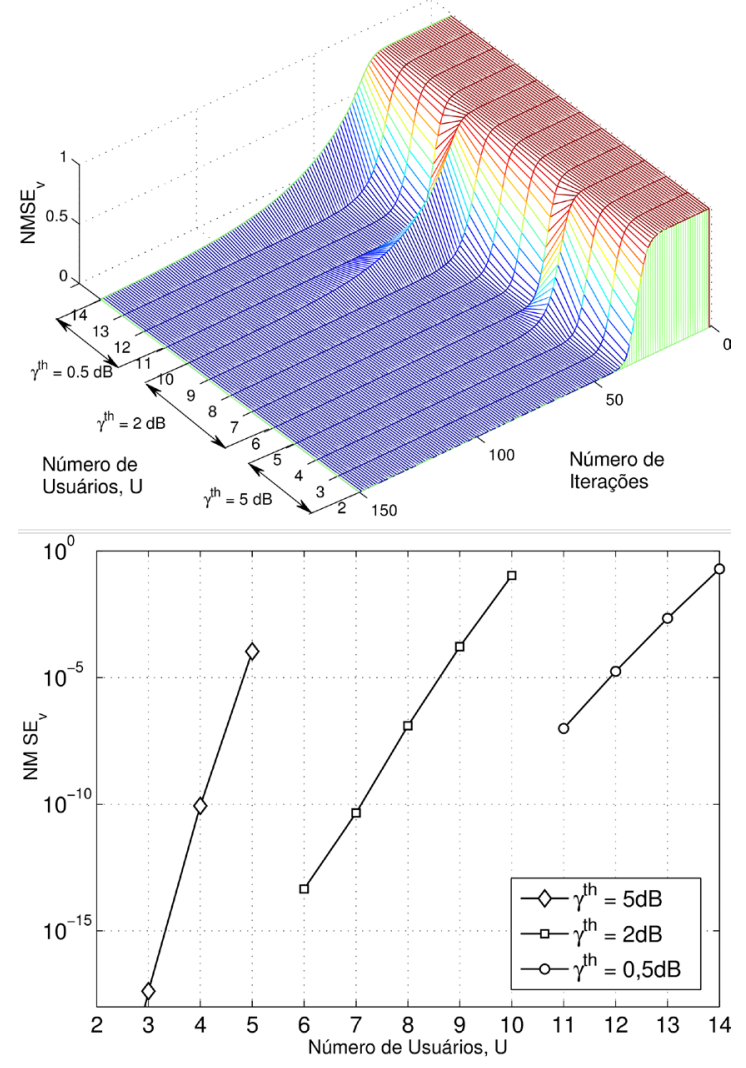

Fonte: Os autores

Da Fig. 1.a, verifica-se que o número de iterações na faixa de I $€[40 ; 150]$ é suficiente para que o PCA de Verhulst atinja qualidade na convergência para a faixa de usuários $U \in[2 ; 14]$ sendo I crescente em função do incremento do número de usuários (MAI crescente). Adicionalmente, para um dado número fixo de iterações, $k=\mathrm{I}=70$ a Fig. 1.b, indica que a qualidade da solução encontrada pelo PCA de Verhulst é função do nível de interferência e da SINR mínima admitida, $\gamma^{\text {th }}$.

\section{Análise de Complexidade}

A análise da complexidade computacional dos algoritmos em questão foi realizada em função do número total de operações de ponto flutuante (floating-point operations ou flop). Assim, foi possível expressar a complexidade como uma função em relação ao número de usuários do sistema. Para isto, foi utilizada a definição de flop em (BOYD; VANDENBERGHE, 2004), onde cada flop representa uma operação de soma, subtração, multiplicação ou divisão entre dois números de ponto flutuante. Adicionalmente, foi incluída a complexidade de execução da versão do algoritmo Analítico, baseado em (7), porém desconsiderando a complexidade introduzida pelo Método da Potência (MP) na determinação da existência da solução ótima (Analítico). Este procedimento foi adotado para se avaliar o impacto da complexidade computacional necessária para se garantir a existência e unicidade do vetor de potência ótimo. A tabela 2 resume a complexidade de cada algoritmo.

Tabela 2 - Complexidade em termos de flops

\begin{tabular}{lc}
\hline Algoritmo & Complexidade \\
\hline Analítico & $5 \mathrm{u}^{3}-\mathrm{u}^{2}+4 \mathrm{u}+2 \sum_{i=1}^{u} i^{2}$ \\
$\begin{array}{l}\text { Analítico } \\
+\mathrm{MP}\end{array}$ & $5 \mathrm{u}^{3}+\left(4 \mathrm{I}_{\text {max }^{-}}-1\right) \mathrm{u}^{2}+\left(7+6 \mathrm{I}_{\max }\right) \mathrm{u}+2 \sum_{i=1}^{u} i^{2}$ \\
Verhulst & $2\left(\mathrm{I}_{\text {max }^{\mathrm{v}}}-1\right) \mathrm{u}^{2}+9\left(\mathrm{I}_{\text {max }}^{\mathrm{v}}-1\right) \mathrm{u}$ \\
\hline
\end{tabular}

$\mathrm{u}=$ número de usuários

Fonte: Os autores

Figura 2 exibe o comportamento dos algoritmos em função do número de usuários do sistema. Observa-se que para um número pequeno de usuários, $U \leq 16$, o PCA-Verhulst apresenta um custo 
computacional superior ao analítico. No entanto, ao aumentar o número de usuários, a sua utilização é justificada por apresentar ordem de complexidade menor, $\mathrm{O}\left(n^{2}\right)$, enquanto que o método Analítico é limitado assintoticamente por $\mathrm{O}\left(n^{3}\right)$. Isto se deve ao alto custo envolvido na multiplicação e na inversão de matrizes, sendo aqui utilizado o método de eliminação de Gauss com pivotamento parcial (PANG, 2006) para a inversão de matrizes, cuja ordem de complexidade resulta $\mathrm{O}\left(n^{3}\right)$ para ambas as operações.

Figura 2 - Operações de ponto flutuante.

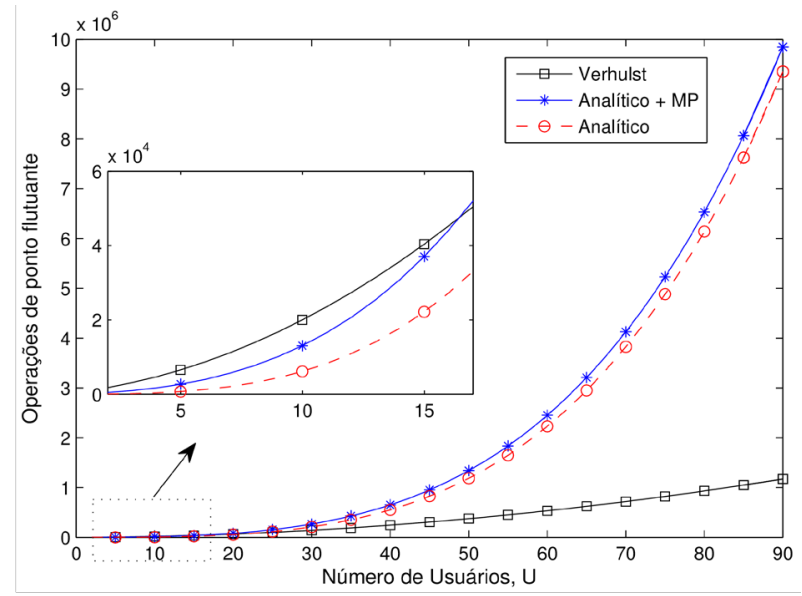

Fonte: Os autores

Com a finalidade de reforçar a análise de complexidade, foram obtidas as médias de ciclos realizados no DSP durante a execução dos algoritmos. O resultado é exibido na Figura 3, considerando número de usuários relativamente reduzido, $U \leq$ 14 . Novamente, observa-se a mesma tendência do aumento de complexidade em função do número de usuários do sistema. Desta forma, por meio da ferramenta cftool do MATLAB, obteve-se por fitting uma equação polinomial para cada algoritmo, a qual descreve o crescimento da complexidade, em termos de número de ciclos executados pelos algoritmos, em função do número de usuários . As equações obtidas encontram-se na Tabela 3
Figura 3 - Ciclos realizados em função do número de usuários.

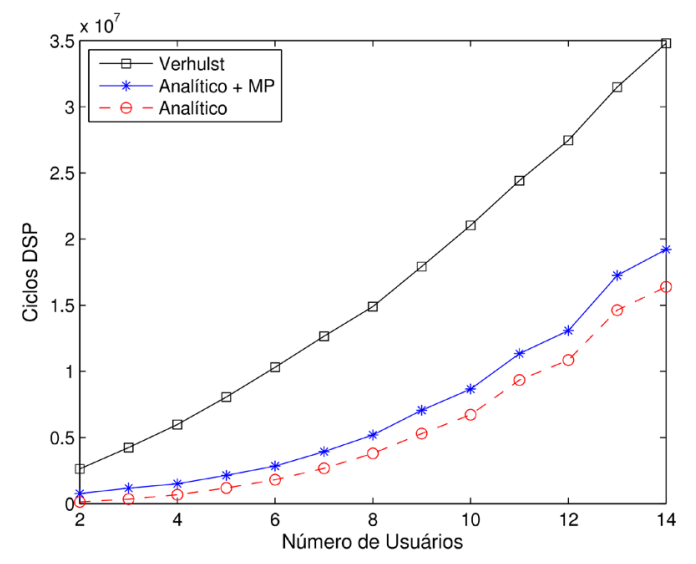

Fonte: Os autores

Tabela 3 - Equações de Número de Ciclos

\begin{tabular}{lc}
\hline Algoritmo & \multicolumn{1}{c}{ Equação } \\
\hline Analítico & $2237 \mathrm{u}^{3}+74250 \mathrm{u}^{2}-318300 \mathrm{u}+539800$ \\
Analítico & $1925 \mathrm{u}^{3}+90440 \mathrm{u}^{2}-327600 \mathrm{u}+1179000$ \\
+ MP & \\
Verhulst & $100600 \mathrm{u}^{2}+1088000 \mathrm{u}+57340$ \\
\hline
\end{tabular}

Fonte: Os autores

Para ter um controle de potência efetivo, sistemas CDMA da segunda geração (IS-95) utilizam uma taxa de atualização na malha de controle de potência igual a 800 atualizações/segundo, o equivalente a uma atualização a cada 1,25 ms. Já em sistemas CDMA de terceira geração (WCDMA), essa taxa de atualização é dobrada, exigindo uma atualização a cada $0,625 \mathrm{~ms}$. A fim de verificar a viabilidade de implementação dos PCAs em plataformas DSP, estimou-se o tempo médio de execução de cada algoritmo a partir da relação:

Tempo de Execução em DSP: $\quad T_{\mathrm{DSP}}=\frac{\text { Número de ciclos }}{\text { Frequência do DSP }}$

Assim, foi possível obter uma estimativa sobre a possibilidade de uso de um DSP, ou um hardware equivalente, em realizar o controle de potência em 
sistemas 2G e 3G CDMA. O resultado encontrase na Fig. 4. Verifica-se que apesar dos tempos de execução dos dois algoritmos resultarem ligeiramente superiores aos tempos máximos estabelecidos pelos $T_{\text {atual }}-2 \mathrm{G}$ e $T_{\text {atual }}-3 \mathrm{G}$, os resultados indicam que o tempo de execução do algoritmo de PCAVerhulst tem um crescimento polinomial quadrático com o número de usuários, o que torna factível a implementação da solução de controle de potência empregando-se plataforma DSP com um poder/ velocidade de processamento ligeiramente superior ao oferecido pela plataforma DSP utilizada neste trabalho. Observa-se ainda comportamento semelhante ao da Figura 2, no entanto, com a inversão do custo computacional ocorrendo com um número superior de usuários (30 usuários), isto se deve em parte pelo método de contagem de flops adotado (BOYD; VANDENBERGHE, 2004), o qual não difere multiplicações de adições e subtrações e também por desconsiderar o custo computacional das outras operações realizadas pelos algoritmos, como atribuições e alocação de memória.

\section{Conclusões}

Este trabalho discute a viabilidade de implementação de dois algoritmos de controle de potência em sistemas DS/CDMA comerciais, particularmente sistemas $2 \mathrm{G}$ e $3 \mathrm{G}$. Como prova-deconceito é quantificado neste trabalho o número de ciclos DSP e o tempo de execução dos PCAs, para um número crescente de usuários, necessários para se atingir o equilíbrio do controle de potência. Resultados numéricos indicam factibilidade de implementação do algoritmo de Verhulst empregando-se uma plataforma com poder/velocidade de processamento marginalmente superior, porém sendo suficiente uma plataforma de mesma capacidade de processamento à empregada neste trabalho. Através da análise de complexidade, demonstra-se também a elevado custo de implementação do método analítico para ambientes reais, exigindo assim métodos alternativos que aproximem o vetor de potência ótimo do sistema.
Figura 4 - Tempo de execução dos PCAs em função do número de usuários. Limites de atualização do controle de potência nos padrões CDMA $2 \mathrm{G}$ e $3 \mathrm{G}$.

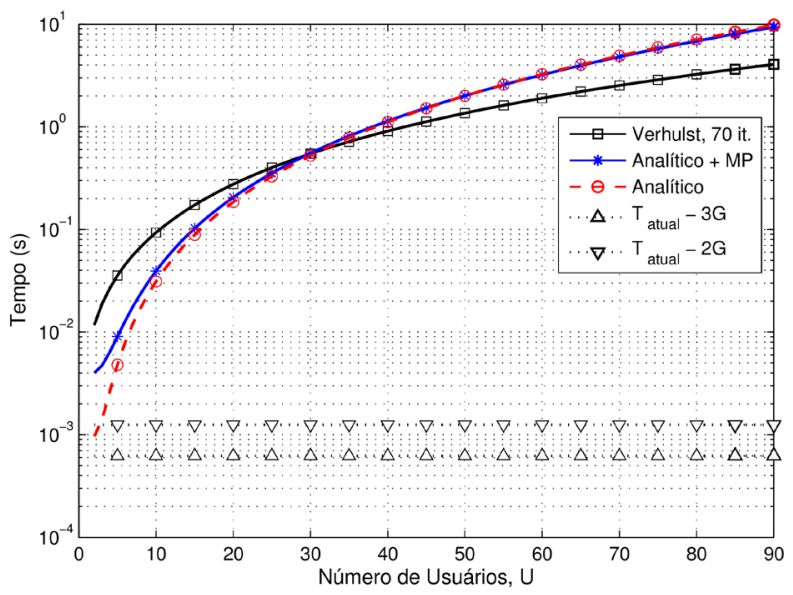

Fonte: Os autores

\section{A Aspectos de Implementação de PCAs.}

\section{A.1 Plataformas de Processamento Digital de Sinais.}

Processadores digitais de sinais (DSPs digital signal processing) (CHASSAING, 2004) são microprocessadores com uma arquitetura otimizada para realização de diversas operações necessárias no processamento de sinal digital (e.g. tarefas numéricas intensivas), com uma ampla gama de aplicações, como nas comunicações e no processamento de voz e imagem.

Processadores de sinais digitais como o TMS320C6713, da família C6X da Texas Instruments, possuem microprocessadores com um tipo de arquitetura específica, baseada na arquitetura VLIW (very-long-instruction-word), e um conjunto de instruções apropriadas para o processamento de sinais. O desenvolvimento de aplicações é feito por meio do DSP Starter Kit (DSK), o qual é composto por:

- Uma placa contendo o processador de ponto flutuante TMS320C6713 (6713).

- O ambiente de desenvolvimento integrado Code Composer Studio (CCS). 
- Cabo USB que realiza a comunicação entre o PC e a placa DSK.

- Fonte de alimentação de 5V para a placa DSK.

A Fig. 5 exibe o DSK utilizado neste trabalho conectado a um computador pessoal.

Figura 5 - DSP starter kit em funcionamento

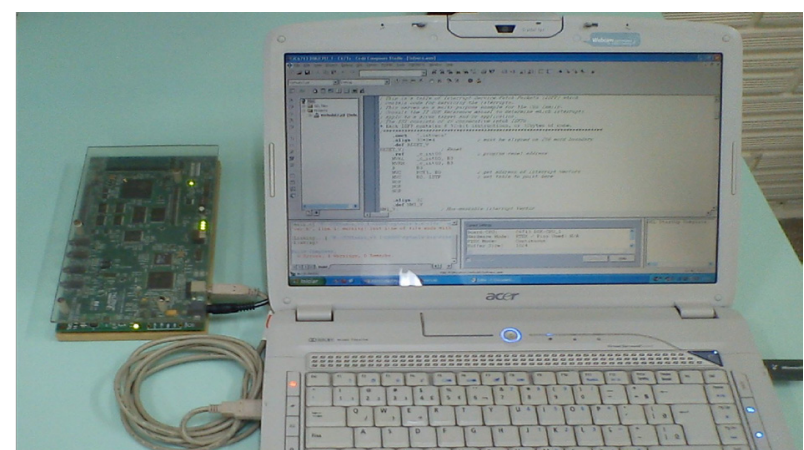

\section{TMS320C6713}

A placa opera na frequência de $225 \mathrm{MHz}$, possuindo 16 Mbytes de memória SDRAM e 512 Kbytes de memória flash (sendo 256 Kbytes utilizadas na configuração padrão). A estrutura da memória interna possibilita que um total de 8 instruções possam ser buscadas a cada ciclo. $\mathrm{O}$ clock de $225 \mathrm{MHz}$ o torna capaz de buscar oito instruções de 32 bits a cada 1/(225 MHZ) ou 4.44 ns (CHASSAING, 2004).

\section{Code Composer Studio (CCS)}

O ambiente de desenvolvimento integrado CCS possibilita o uso da linguagem de alto nível C para construção de softwares que executem no DSP. Para isto, conta com um compilador C, capaz de compilar um arquivo .c e produzir um arquivo .asm de código Assembly. O assembler, presente no CCS, converte o arquivo produzindo um objeto de linguagem de máquina com extensão .obj. Por fim, o linker combina o arquivo .obj com as bibliotecas de objetos para produzir um arquivo com extensão .out, que pode ser carregado e executado diretamente no DSP.

O CCS ainda possibilita a troca de dados em tempo real com outros softwares, como o MATLAB da empresa MathWorks, através da tecnologia realtime data exchange (RTDX).

\section{B Teorema de Perron-Frobenius.}

\section{B1 Autovalores e autovetores.}

Temos por raio espectral da matriz A representado por $\rho(\mathbf{A})$, o maior valor absoluto dentre os autovalores de $\mathbf{A}$. De forma que os autovalores e autovetores associados a uma matriz A, devem respeitar a seguinte igualdade (PILLAI; SUEL; CHA, 2005).

$$
\mathbf{A x}=\lambda \mathbf{x}
$$

Onde $\mathbf{X}$ e $\lambda$ representam os autovetores $\mathrm{e}$ autovalores, respectivamente. Assim o raio espectral pode ser definido como

$$
\rho(\mathbf{A})=\max _{i}\left|\lambda_{i}(\mathbf{A})\right|
$$

\section{B2 Teorema de Perron-Frobenius.}

O teorema de Perron-Frobenius postula que uma matriz quadrada não negativa e regular apresentará um único autovalor, maior em magnitude do que os demais (raio espectral), com um autovetor correspondente que possui entradas estritamente positivas.

Teorema 1 (Teorema de Perron-Frobenius). Seja A uma matriz $n \times n$ não negativa e regular, então $\mathbf{A}$ possui ao menos um autovalor $\lambda_{p}$ com as seguintes propriedades: (SENETA, 2006)

a) $\lambda_{p}>0$

b) Os autovetores, esquerdo e direito, associado $a \lambda_{p}$ são positivos.

c) Se $\lambda_{p}$ é outro autovalor de $\mathbf{A}$, então $\lambda_{p}>|\lambda|$. 
d) Os autovetores associados a $\lambda_{p}$ são únicos para múltiplas constantes.

e) $S e 0 \leq \mathbf{B} \leq \mathrm{T}$ e $\beta$ é um autovalor de $\mathbf{B}$, então $|\beta| \leq \lambda_{\mathrm{p}}$. Além disso, $|\beta|=\lambda_{\mathrm{p}}$ implica que $\mathbf{B}=\mathbf{A}$.

f) $\lambda_{\mathrm{p}}$ é uma raíz simples da equação característica de $\mathbf{A}$.

Para uma matriz $\mathbf{A}$ irredutível e não negativa todas as declarações contidas no Teorema 1 mantém-se verdadeiras, exceto a afirmação (c), que é substituída por: Se $\lambda_{\mathrm{p}}$ é outro autovalor de $\mathbf{A}$, então $\lambda_{\mathrm{p}} \geq|\lambda|$.

A generalização dos aspectos da estrutura do teorema de Perron-Frobenius para matrizes não negativas e irredutíveis permite a obtenção de garantias de existência de soluções estritamente positivas $(\mathbf{x} \succ 0)$ para a equação a seguir, relevante na área econômica e na análise numérica (SENETA, 2006).

$$
(s \mathbf{I}-\mathbf{A}) \mathbf{x}=\mathbf{b}
$$

Teorema 2. A condição necessária para existência de uma solução $\mathbf{x}$, para qualquer $\mathbf{b} \geqslant 0$ é que seja maior que o raio espectral de $\mathbf{A}$. Neste caso haverá somente uma solução positiva dada por

$$
\mathbf{x}=(s \mathbf{I}-\mathbf{A})^{-1} \mathbf{b}
$$

Deste modo, para a equação (7), onde $\mathrm{s}=1$, existirá solução se e somente se $\rho(\mathbf{A})<1$. A prova deste teorema pode ser obtida em (SENETA, 2006).

\section{Referências}

BAPAT, R.; RAGHAVAN, T. Non-negative matrices and applications. Cambridge: Cambridge University Press, 1997.

BOYD, S.; VANDENBERGHE, L. Convex optimization. Cambridge: Cambridge University Press, 2004.

CHASSAING, R. Digital signal processing and applications with the C6713 and C6416 DSK. Hoboken: Wiley-Interscience, 2004.
DONMEZ, N. A game-theoretic approach to efficient power control in cdma data networks. In: INTERNATIONAL SYMPOSIUM ON INNOVATIONS IN INTELLIGENT SYSTEMS AND APPLICATIONS, 2011, Istanbul. Proceedings... Istanbul: INISTA, 2011. p. 248-252.

GOODMAN, D. J.; MANDAYAN, N. B. Power control for wireless communication. IEEE Personal Communication Magazine, New York, v. 7, n. 4, p. 4854, Apr. 2000.

GROSS, T. J.; ABRÃO, T.; JESZENSKY, P. J. E. Modelo populacional de verhulst aplicado ao controle de potência distribuído ds/cdma. Semina: Ciências Exatas e Tecnológicas, Londrina, v. 26, n. 1, p. 83-100, jan./jun. 2005.

GROSS, T. J.; ABRÃO, T.; JESZENSKY, P. J. E. Distributed power control algorithm for multiple access systems based on verhulst model. International Journal of Electronics and Communications, Wiesbaden, v. 65, n. 4, p. 361-372, 2010.

HAYKIN, S.; MOHER, M. Sistemas de comunicações wireless. São Paulo: Bookman, 2008.

MIAO, G.; HIMAYAT, N.; LI, Y. Energy-efficient link adaptation in frequency-selective channels. IEEE Transactions on Communications, New York, v. 58, n. 2, p. 545-554, Feb. 2010.

PANG, T. An introduction to computational physics. Cambridge: Cambridge University Press, 2006.

PILLAI, S.; SUEL, T.; CHA, S. The perron-frobenius theorem: some of its applications. IEEE Signal Processing Magazine, v. 22, n. 2, p. 62-75, Mar. 2005.

RODRIGUEZ, V. An analytical foundation for resource management in wireless communication. In: GLOBAL TELECOMMUNICATIONS CONFERENCE, 2003, Cagayan de Oro. Proceedings... Cagayan de Oro: IEEE, 2003. v. 2, p. 898-902.

SALKUYEH, D. K.; TOUTOUNIAN, F. Optimal iterate of the power and inverse iteration methods. Applied Numerical Mathematics, Amsterdam, v. 59, n. 7, p. 15371548, 2009.

SAMPAIO, L. D. H. Alocacão de potência e taxa de informação em redes sem fio de múltiplo acesso. 2010. Dissertação (Mestrado em Ciência da Computação) Universidade Estadual de Londrina, Londrina.

SARAYDAR, C.; MANDAYAM, N.; GOODMAN, D. Efficient power control via pricing in wireless data networks. IEEE Transactions on Communications, New York, v. 50, n. 2, p. 291-303, 2002. 
SENETA, E. Non-negative Matrices and Markov Chains. London: Springer, 2006. (Springer Series in Statistics).

SOUZA, A. R. C.; SAMPAIO, L. H.; ABRÃO, T.; JESZENSKY, P. J. E. Energy and spectral efficiencies trade-off with filter optimization in multiple access interference-aware networks. In: SIMPÓSIO BRASILEIRO DE REDES DE COMPUTADORES E SISTEMAS DISTRIBUÍDOS, 30., 2012, Ouro Preto. Anais... Ouro Preto: SBRC, 2012. v. 1. p. 1-14.

ULlAH, M.; RAIHAN, S.; BARY, M. Performance analysis of power control schemes in cdma communication system. In: INTERNATIONAL CONFERENCE ON MECHATRONICS, 4., 2011, Kuala Lumpur. Proceedings... Kuala Lumpur: ICOM, 2011. p. $1-4$.

ULUKUS, S.; YATES, R. Stochastic power control for cellular radio systems. IEEE Transactions on Communications, New York, v. 46, n. 6, p. 784-798, Jun. 1998.

VERHULST, P. F. Notice sur la loi que la population poursuit dans son accroissement. Correspondance Mathématique et Physique, Bruxelles, v. 10, p. 113-121, 1838.

Recebido em 27 Março 2012-Received on March 27, 2012.

Aceito em 4 Junho, 2012 - Accepted on June 4, 2012. 
